\title{
A microfluidic system utilizing molecularly imprinted polymer films for amperometric detection of morphine
}

\author{
Chen-Hsun Weng ${ }^{\mathrm{a}}$, Wei-Ming Yeh ${ }^{\mathrm{b}}$, Kuo-Chuan $\mathrm{Ho}^{\mathrm{b}}$, Gwo-Bin Lee ${ }^{\mathrm{a}, *}$ \\ a Department of Engineering Science, National Cheng Kung University, Tainan, Taiwan \\ ${ }^{\mathrm{b}}$ Department of Chemical Engineering, National Taiwan University, Taipei, Taiwan
}

Received 14 November 2005; received in revised form 23 April 2006; accepted 24 April 2006

Available online 12 June 2006

\begin{abstract}
This study reports a microfluidic system for detecting morphine (MO) using a combination of a molecularly imprinted polymer (MIP) and electrochemical sensing techniques. A monomer, called 3,4-ethylenedioxythiophene (EDOT), was used to mix with morphine molecules through an electropolymerization process on a sensing electrode. The modified MIP-PEDOT (poly-ethylenedioxythiophene as the imprinting polymer with MO as the template) electrode was then used for detecting the morphine via the amperometric method. Key components including MIP films, a PDMS (polydimethylsiloxane)-based microchannel, a peristaltic micropump, microvalves and sensing microelectrodes were integrated to form a new microfluidic system for morphine sensing utilizing MEMS (micro-electro-mechanical-systems) technologies. The morphine samples were automatically transported to the MIP-PEDOT sensing electrode using the peristaltic micropump. Then, the morphine was detected using the electrochemical method. Experimental data show that the sensitivity of the MIP-PEDOT morphine sensor is $171.5 \mu \mathrm{A} / \mathrm{cm}^{2} \mathrm{mM}$ in detecting morphine concentration ranging from 0.01 to $0.2 \mathrm{mM}$ at a flow rate of $92.3 \mu \mathrm{l} / \mathrm{min}$. The novel combination of microfluidics, MIP, and electrochemical sensing technologies provides a promising approach for highly sensitive, highly selective morphine sensing with a low sample consumption rate. More importantly, the whole process can be performed in an automatic format by using the enabling microfluidic technology. A multi-functional electrochemical detection system is feasible using similar microfluidics/MIP/electrochemical technologies.
\end{abstract}

(c) 2006 Elsevier B.V. All rights reserved.

Keywords: Molecularly imprinted polymer; Microfluidics; Micropumps; MEMS; Morphine; Electrochemical; Amperometric detection

\section{Introduction}

Many methods to detect morphine (MO) have previously been developed, including gas chromatography (GC) [1], liquid chromatography (LC) [2], high performance liquid chromatography (HPLC) [3], ultraviolet (UV) spectroscopy [4], GC-mass spectroscopy (GC-MS) [5], fluorimetry [6], chemiluminescence [7], surface plasmon resonance (SPR) [8], and electrochemical methods [9]. The morphine molecules are usually purified using liquid-liquid extraction or solid phase extraction [10]. Even though chromatography and GC-MS are well-developed methods for morphine detection with a low detection limit, the bulky and expensive apparatus still hinder their practical applications. There still remains a great need for a fast and user-friendly device for morphine sensing.

\footnotetext{
* Corresponding author. Tel.: +886 6 2757575/63347; fax: +886 62761687

E-mail address: gwobin@mail.ncku.edu.tw (G.-B. Lee).
}

Alternatively, immunoassays (IA) which utilize antibodyantigen affinity for detecting bio-samples are another popular method for morphine detection [11]. Enzyme-linked immunosorbent assay (ELISA) is one of the most popular methods for these immunoassays [12]. With this approach, the sample preparation is much simpler and more convenient. More importantly, it is a cost effective method for morphine sensing. However, it is a labor-intensive process and its relatively low accuracy may hinder its practical applications. Test papers made by the GC and the immunochromatography methods have been reported with a detection limit of $300 \mathrm{ng} / \mathrm{ml}$ [13]. However, the range of morphine concentration in human beings changes greatly. For example, the concentration of morphine ranges between 8 and $80 \mathrm{ng} / \mathrm{ml}$ when curing [3] and the concentration of morphine in the urine would also change with time [14]. Thus the major shortcoming for these easy testing methods is their relatively high detection limits and low selectivity. 
Morphine also can be detected using electrochemical methods. Among them, amperometric detection is promising for a portable and fast morphine sensor since it provides current outputs in response to morphine concentrations $[15,16]$. In vivo detection can also be performed using the amperometric method [9]. The amperometric detection of morphine is basically achieved by electrocatalytic oxidation. Such a process is also compatible with HPLC [17] and MIP [18] techniques with high sensitivity and selectivity. Modified electrodes are usually adopted for electrochemical sensing. After modification, the selectivity and sensitivity of the sensing device can be significantly improved. As far as morphine detection is concerned, it was reported that a cobalt hexacyanoferrate-modified glassy carbon electrode can implement the electrocatalytic oxidation of morphine with high stability [9]. Morphine sensing at a Prussian blue (PB)-modified electrode was also reported [19]. In this case, PB acts as an artificial peroxidase of morphine. However, the fabrication process is complicated and timeconsuming.

Another issue for morphine sensing is the selectivity of the sensing methods. The compositions of bio-samples such as urines, serums, and body fluids are complicated. The developed method must have a high selectivity for morphine sensing. MIP has been known to have a high selectivity for bio-sensing [20]. Alternatively, morphine MIP films can be formed using a polymerization process. The performance of MIP films has been extensively investigated [21]. However, these morphine MIP films have not yet been applied for morphine sensing. The current research group has also reported that MIP films could be used for electrochemical detection of morphine [22].

There are several popular methods to fabricate chemically modified electrodes, including adsorption, covalent binding, carbon paste electrodes, and polymerization. Recently, the MIPmodified electrode has been reported and integrated with the electrochemical techniques, such as amperometry, potentiometry, voltammetry, and conductometry [23]. Since MIP has a high selectivity to specific samples, MIP-modified electrodes have attracted considerable interests.

A cheap, user-friendly, highly sensitive, highly selective method is still in great demand for morphine sensing. The conducting polymer, poly(3,4-ethylenedioxythiophene) (PEDOT), was previously utilized to prepare the MIP-PEDOT thin film at the ITO electrode to enhance the selectivity of the modified electrode in detecting morphine [22]. In this study, a MIP-PEDOT film formed on a Pt electrode was acted as the recognition site with a high selectivity in combination with an electrochemical transducer. Besides, a microfluidic system capable of transporting a small amount of bio-samples has been used for automation of sensing process. MEMS and microfluidic techniques have enabled the miniaturization of biochemical devices and systems. The advantages of microfluidic analytical and detection systems include high sensitivity, disposability, low reagent and sample consumption, portability, low power consumption, and low cost. Significantly, the functionality and reliability of micromachined biochemical devices can be improved by integrating mature integrated circuits (IC) technology with various microfluidic devices to form a micro-total-analysis-system [24].

\section{Materials and methods}

\subsection{Design}

In this study, a MIP film was formed on the surface of sensing electrodes to enhance the selectivity of sensing. An electrochemical method was then used for detection of morphine. An electro-polymerization process was used to form the MIP films on the surface of electrodes directly. The fabrication process is simple and easy, and the thickness of the membrane is accurately controllable to the nanometer level [25]. Fig. 1(a) shows a schematic diagram of the microfluidic MIP-morphine sensing system. The sample injection can be automated by using micropumps and microvalves. Samples with different concentrations can be injected sequentially from either reservoir. The purpose of the buffer reservoir is for washing. A MIP-morphine electrode was integrated into the microfluidic chip. The sample flow passing through each reaction region was kept uniform by using a "spider-web" micropump developed by the current research group [24]. Three electrodes consisting of a Pt working electrode, a Pt counter electrode, and a silver $(\mathrm{Ag})$ reference electrode [26] were used for electrochemical sensing of morphine, as shown in Fig. 1(b).

\subsection{Fabrication}

The microfluidic MIP-morphine sensing system was composed of three essential components. The first one was the microelectrodes fabricated using thin-film deposition techniques. The second one was the MIP-morphine film made by electropolymerizing a PEDOT film on the Pt electrode. The last one was the microfluidic system, including microchannels, micropumps and microvalves, which were fabricated using MEMS technology.

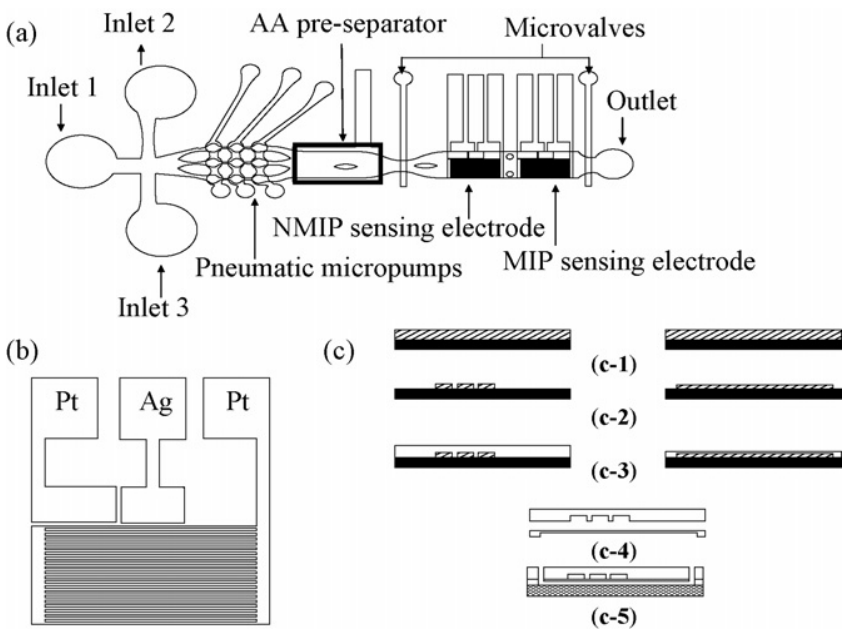

Fig. 1. (a) A schematic diagram of the microfluidic MIP-morphine sensing system with a combination of microfluidics, MIP and electrochemical sensing electrodes. (b) The sensing zone includes the working electrode $(\mathrm{Pt})$, the reference electrode $(\mathrm{Ag})$, and the counter electrode $(\mathrm{Pt})$. (c) Fabrication process for double-layer PDMS fluid control devices. (c-1) SU-8 spin-coating, (c-2) photolithography, (c-3) PDMS casting, (c-4) PDMS de-molding, (c-5) PDMS bonding. 


\subsubsection{Fabrication of microelectrodes}

Microelectrodes were fabricated by using a standard photolithography process. First, a Pt (1000 ̊)/titanium (Ti, $300 \AA)$ electrode with a dimension of $1.45 \mathrm{~cm} \times 0.57 \mathrm{~cm}$ was deposited and patterned as a pre-separator on the glass substrate using an E-beam evaporation process, followed by a standard lift-off process. Meanwhile, three microelectrodes, including a working electrode, a reference electrode and a counter electrode, were designed to sense morphine. Note that the morphine-MIP was formed on the working electrode by using an electropolymerization process. The working electrode and the counter electrode were fabricated by depositing and patterning a $\mathrm{Pt}$ (1000 $\AA$ ) layer with an adhesion layer of Ti (300 ̊). The reference electrode was fabricated by depositing and patterning $\mathrm{Ag}$ $(1000 \AA) / \mathrm{Au}(300 \AA) / \mathrm{Ti}(300 \AA)$ layers. Note that the Au/Ti layers were used to promote adhesion. The electrodes were formed using similar photolithography and E-beam evaporation processes as described above. Finally, the microelectrodes were immersed in a $0.1 \mathrm{M} \mathrm{FeCl}_{3}$ solution for $2 \mathrm{~min}$ to generate an oxidation-reduction reaction on the $\mathrm{Ag}$ electrode forming $\mathrm{AgCl}$ as the $\mathrm{Ag} / \mathrm{AgCl}$ reference electrode.

\subsubsection{Fabrication of MIP films}

The fabrication process of MIP films involves three steps, including combination, polymerization, and extraction [15]. The monomer and the template were first formed as a complex. Then macroporous sites of microspheres were formed by using monomer polymerization or cross-linkers. Finally, the template was extracted to form molecularly imprinted polymer films. The MIP-PEDOT modified electrode was prepared by electropolymerizing an EDOT monomer onto the working electrode in an acetonitrile $(\mathrm{MeCN})$ solution in which 0.01 M EDOT monomer, $0.1 \mathrm{M}$ lithium perchlorate $\left(\mathrm{LiClO}_{4}\right)$, and morphine molecules were dissolved. Prior to the electropolymerization, the solution was deoxygenated by aerating with nitrogen. A cyclic voltammetry method was used to deposit MIP-PEDOT films onto the working electrode. After electropolymerization, the surface of the MIP-PEDOT modified electrode was washed with MeCN. $\mathrm{MeCN}$ was then volatilized in an air exhauster. After soaking the modified electrodes in deionized water (DIW), the morphine trapped in the modified electrodes was removed by methanol. Cyclic voltammetric scanning was performed in $0.1 \mathrm{M} \mathrm{KCl}$ every $10 \mathrm{~min}$ to verify that a morphine reaction was not observed, indicating that morphine has been completely extracted. By preparing the MIP-PEDOT modified electrode, morphine was imprinted and specific reaction sites were formed in the working electrode.

Two sets of microelectrodes were designed for detecting morphine. The first set of microelectrodes was designed to adsorb AA (ascorbic acid) as a pre-separator since the AA is known to have a high interference for morphine detection [27]. Thus the usage of a pre-separator electrode to adsorb AA could enhance the performance of the morphine sensor. In this study, an AA Nylon-MIP film was coated upstream of the microchannels as a pre-separator to adsorb AA. The molecularly imprinted Nylon-6 film was prepared by the phase inversed method [28]. Nylon-6 (20 wt.\%) and ascorbic acid (8 wt.\%) were dissolved in formic acid at $50{ }^{\circ} \mathrm{C}$ and cast on the microelectrode substrate in the pre-separator region. After the formation of the Nylon- 6 film at room temperature, the AA MIP-Nylon 6 was immersed in DIW to wash out the AA template batch-wise for $30 \mathrm{~min}$ and the extraction procedure was repeated for five times. Non-imprinted polymer (denoted as AA NMIP-Nylon 6) was prepared on another microelectrode substrate in the pre-separator region and obtained in the same way except that the AA template was not added in the re-solidification of the Nylon-6 film.

The second set of microelectrodes was designed to detect morphine by electropolymerizing a PEDOT film on the $\mathrm{Pt}$ electrode. To better understand the specific sites of $\mathrm{MO}$ on the MIP-PEDOT layer, the reference layer (NMIP-PEDOT layer) was prepared on a separate working electrode which was placed in the upstream of the MIP-PEDOT electrode, as seen in Fig. 1(a). The NMIP-PEDOT electrode was obtained in the same way as that of the MIP-PEDOT electrode except that the morphine template was not added in the electropolymerization procedure.

\subsubsection{Fabrication of microfluidic systems}

The microfluidic device was fabricated by using PDMS (SilMore Industrial Ltd., Sylgard 184A and Sylgard 184B, USA) casting methods to produce inverse structures of microfluidic devices from a SU-8 thick-photoresist mold on a silicon substrate [24]. As shown in Fig. 1(c), two SU-8 molds were first formed, one for the air control channels and another for the microfluidic channels. In order to generate the mold masters, thick photoresist (SU-8-50, MicroChen, Newton, MA, USA) was patterned on a Si wafer by using a photolithography process. The microfluidic channel layer was obtained by spin-coating the PDMS at $700 \mathrm{rpm}$ for $30 \mathrm{~s}$ on the corresponding mold. The PDMS curing process was performed at $95^{\circ} \mathrm{C}$ for $120 \mathrm{~min}$. The thick PDMS layer was then mechanically peeled off from its mold. The two PDMS layers were bonded together using an oxygen plasma treatment. Finally, the PDMS structures and the glass substrate containing sensing electrodes were aligned and bonded at $90^{\circ} \mathrm{C}$ for $20 \mathrm{~min}$. A microfluidic device that combines the microchannels with the micropumps and the microvalves is shown in Fig. 2. The dimensions of the chip are $4.5 \mathrm{~cm} \times 11.5 \mathrm{~cm}$.

\subsection{Experimental setup}

The control system for the MIP-morphine sensing chip was composed of a control circuit, five electromagnetic valve (EMV) switches (SMC Inc., S070M-5BG-32, Japan), and an air compressor (JUN-AIR Inc., MDR2-1A/11, Japan). An 8051 microcontroller (AT89C51, ATMEL, USA) was used to provide signals to control the five EMV switches so that compressed air could be injected into appropriate air channels to activate peristaltic micropumps. The pumping rate of the micropumps was regulated by adjusting the operating frequency of the EMVs and the applied pressure of the compressed air [24]. The valving effect was achieved by providing compressed air to completely shut off the flow channel. A potentiostat (Model 263A, EG\&G Instruments, USA) was used to acquire amperometric signals from the sensing electrodes. The electrical signals were then 


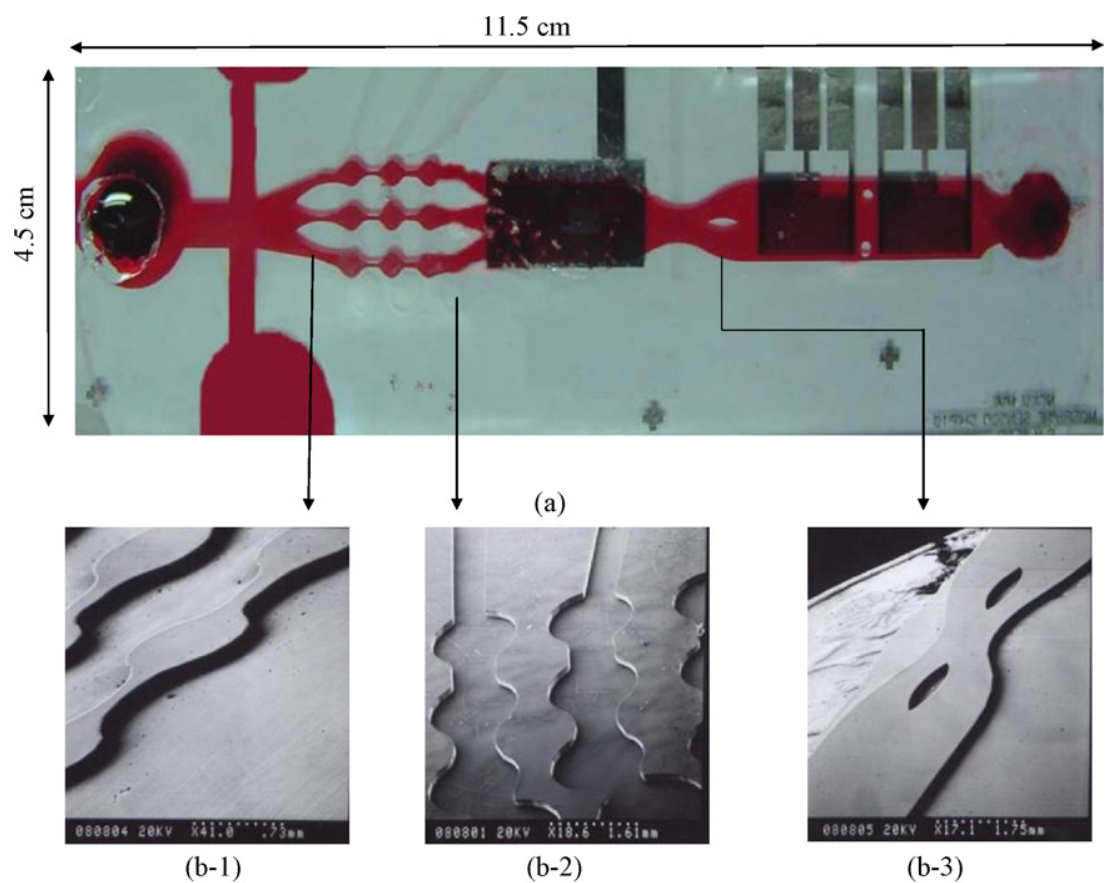

(b)

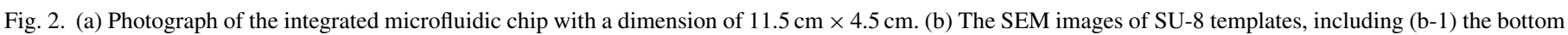
of fluid-pumping zone, (b-2) the air control channel, and (b-3) the fluidic channel.

converted into analog signals and acquired by a commerciallyavailable dual-channel 24-bit ADC module (Model 0224-2, SISC, Taipei, Taiwan) using a personal computer.

\section{Results and discussion}

The $\mathrm{Pt}$ electrode modified by electropolymerization of PEDOT could easily oxidize morphine at a lower potential and achieve a higher current response. It indicated that the PEDOT could serve as an electrocatalyst for morphine oxidation. Therefore, we utilized PEDOT to prepare the MIP-PEDOT thin film at the Pt electrode to enhance the selectivity of the modified electrode in detecting morphine. The oxidation of morphine in aqueous solution has been studied extensively. The oxidation of morphine could be considered to occur in two steps; one arose from a one-electron oxidation of the phenolic group, followed by another one-electron loss from the oxidation of pesudomorphine. The oxidation of morphine could be described by the mechanism as described as follows [22]. Morphine oxidation could occur on the MIP-PEDOT modified electrode before the template was extracted. After template extraction, the MIP-PEDOT modified electrode revealed the capacitive feature of PEDOT. As PEDOT films oxidized morphine at a lower potential than that on the Pt electrode and exhibited a higher current response, one could fabricate a morphine sensor with the PEDOT film based on the molecular imprinting technique. Before amperometric detection, the operating potential was determined using a polarization curve. The net steady-state current was obtained by subtracting the background current of $0.1 \mathrm{M} \mathrm{KCl}$ from the current of a sample containing $1 \mathrm{mM}$ morphine at each potential. Based on the polarization curve, the plateau between 0.40 and $0.50 \mathrm{~V}$ was identified as the limiting current zone, which was generated from mass transport. The operating potential was thus set at $0.45 \mathrm{~V}$. By applying the potential within this range, the current recorded was proportional to the morphine concentration. In general, amperometric detection at steady state with limited current outputs led to the most-reliable analysis. Amperometric measurement could be carried out to examine the current responses for the MIP-PEDOT modified electrode.

The performance of the micropumps was first investigated. Briefly, the pneumatic pump was composed of three PDMS membranes driven by three EMVs. The compressed air filled up the cavities formed from PDMS membranes ordinally such that the solutions could be pushed forward. The individual membranes were deflected peristaltically by the compressed air by adjusting the frequency of the EMV and the applied pressure of the compressed air. Hence, the samples were driven in a specific direction. The pneumatic spider-web micropumps were based on the peristaltic effect generated by resilient polymer membranes and provided uniform flow with a higher pumping rate. The "spider-web" pump was composed of an array of PDMS membranes with a layout similar to a "spider-web". Notably, the spider-web micropump was designed to simultaneously pump the fluids through multiple microchannels. More detail information could be found in our previous work [24]. Fig. 3 shows the pumping rates obtained from the pneumatic micropump at different frequencies. It was observed that the pumping rates could be precisely controlled by changing the driving frequency. The micropump could continuously drive fluids into the microchannel. The maximum pumping rate obtained was $92.3 \mu \mathrm{l} / \mathrm{min}$ at a pressure of $10 \mathrm{psi}$ and a driving frequency of $35 \mathrm{~Hz}$. The pumping rate also depended on the applied pressure. 


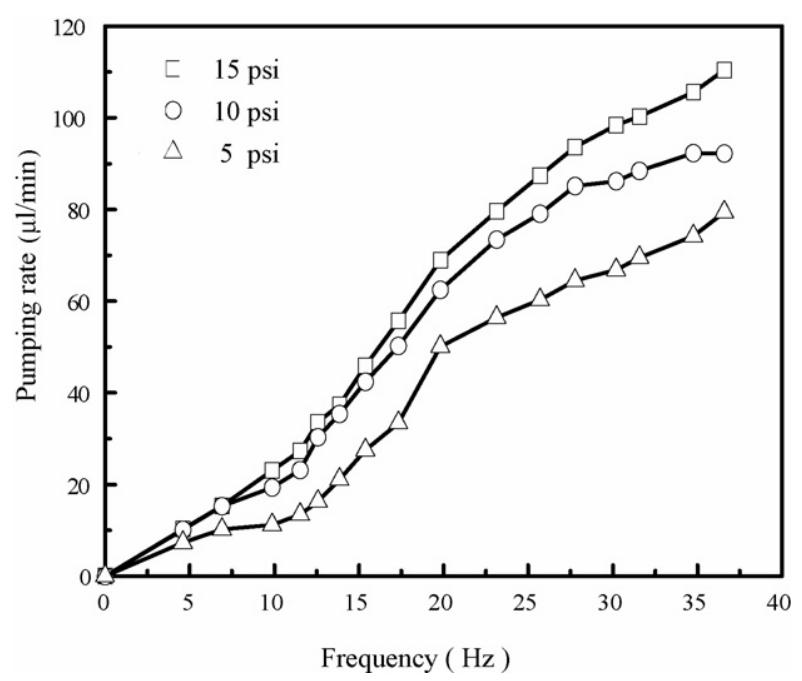

Fig. 3. The relationship between pumping rate and driving frequency for the peristaltic pneumatic micropump.

As the applied pressure increased, the pumping rate increased accordingly.

The microvalve was also tested. As shown in Fig. 4, the microvalve could block fluid flow completely. The deflection of the PDMS membrane ensured that no fluid flew in the "closed" state. Three flow rates (30, 60 and $90 \mu \mathrm{l} / \mathrm{min}$ ) were tested. Experimental data showed that 9 psi was required to block the fluid flow.

The mass transfer of the morphine sample to the sensing electrode plays an important role in this type of electrochemical sensing device. Thus the effect of sample pumping rates on the sensing device was explored. Different pumping rates at different EMV switches driving frequencies did not change appreciably the magnitude of the output signals. However, this had enormous influence on the noise level from the morphine sensing system. When the driving frequency of the pneumatic micropump was low, the flow rate was relatively slow and the

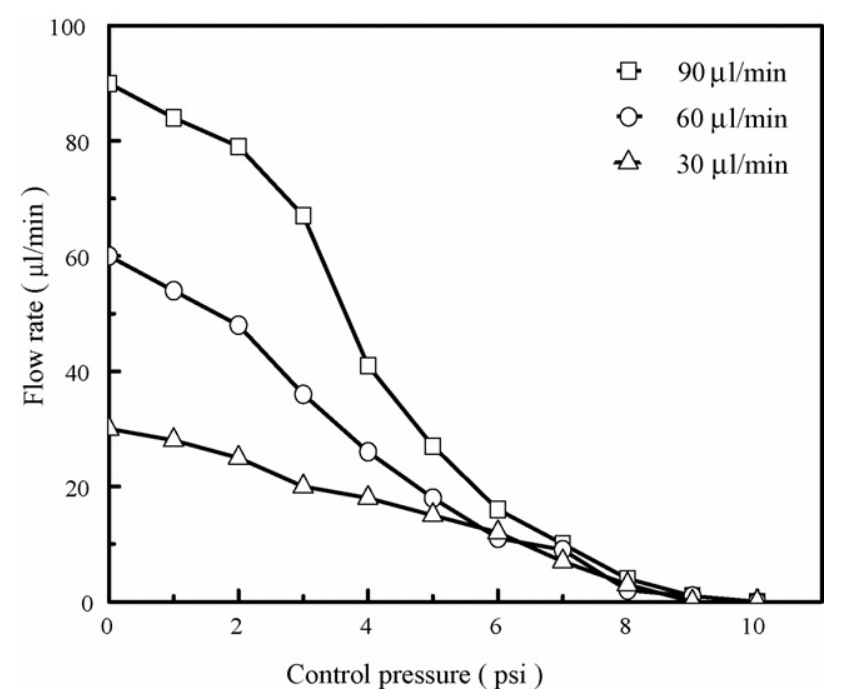

Fig. 4. The relationship between flow rates and control pressures applied on the micro pneumatic valve. time interval for any given slug of fluid to traverse the distance between two membranes of the pneumatic pump was relatively long. The step-wise flowing and stopping motion of the fluids was obvious. Thus perturbation of the fluid was apparent, resulting in a higher noise level. If the fluid was pumped at a higher flow rate at a higher driving frequency, the traverse time interval between two membranes was shorter. A nearly continuous motion of the fluid was generated. It could be seen that the perturbation of the fluid would be smaller and the noise level was lower, accordingly. Thus it is found that a higher signal-to-noise level existed for higher pumping rates. In this study, a driving frequency of $35 \mathrm{~Hz}$ was chosen for morphine testing with a corresponding flow rate of $92.3 \mu \mathrm{l} / \mathrm{min}$ at a pressure of $10 \mathrm{psi}$.

When the applied potential was set at $0.45 \mathrm{~V}$, which was the potential between the working electrode and the reference electrode, the oxidative current of MO was observed. The magnitude of the detected current was dependent on the mass flow rate at which morphine diffused to the surface of the sensing electrodes inside the microchannel. When the MO concentration was low, its response current density was low. Correspondingly, when the MO concentration was increased, the mass flow rate became higher and its response current density increased accordingly. Fig. 5 shows the transient current densities in response to morphine oxidation at $0.45 \mathrm{~V}$ with a step-wise changed in concentration. The steady-state current densities of the MIP-PEDOT modified electrode were reached after adding $0.01,0.025,0.05,0.075,0.10$, and $0.20 \mathrm{mM}$ morphine. The current density returned to its original value after injecting the phosphate buffered saline (PBS), indicating the reversibility of the microsystem. Thus, the net current densities on the MIP and NMIP microelectrodes were acquired over the morphine concentration range from 0.01 to $0.20 \mathrm{mM}$. The sensitivities for the MIP-PEDOT and NMIP-PEDOT electrodes were found to be 171.5 and $144.0 \mu \mathrm{A} / \mathrm{cm}^{2} \mathrm{mM}$, respectively. It was found that the current density response of the MIP-PEDOT electrode was higher than that of the NMIP-PEDOT electrode. This implied

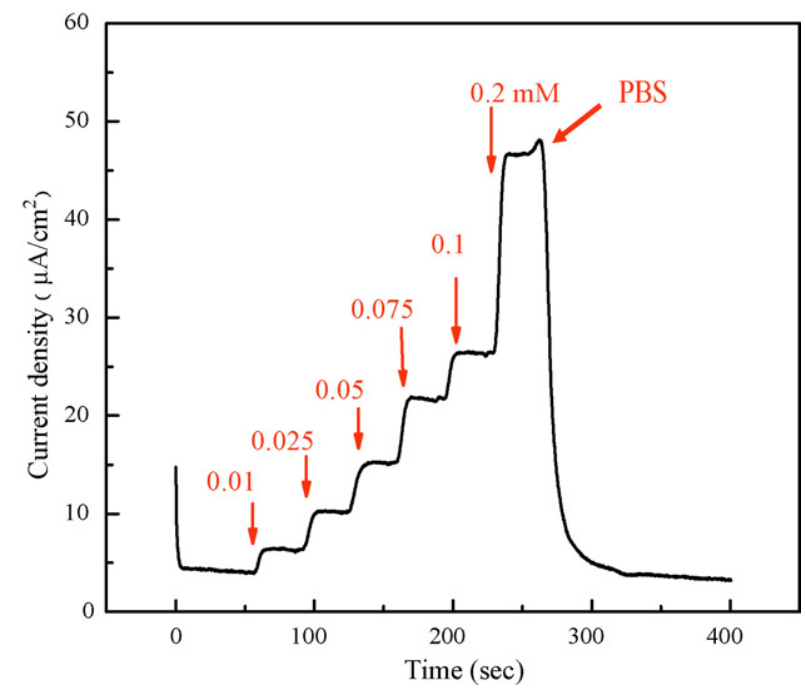

Fig. 5. The transient current densities in response to a step-wise increase of morphine concentration. The applied potential is $0.45 \mathrm{~V}$ and the flow rate is $92.3 \mu \mathrm{l} / \mathrm{min}$. 


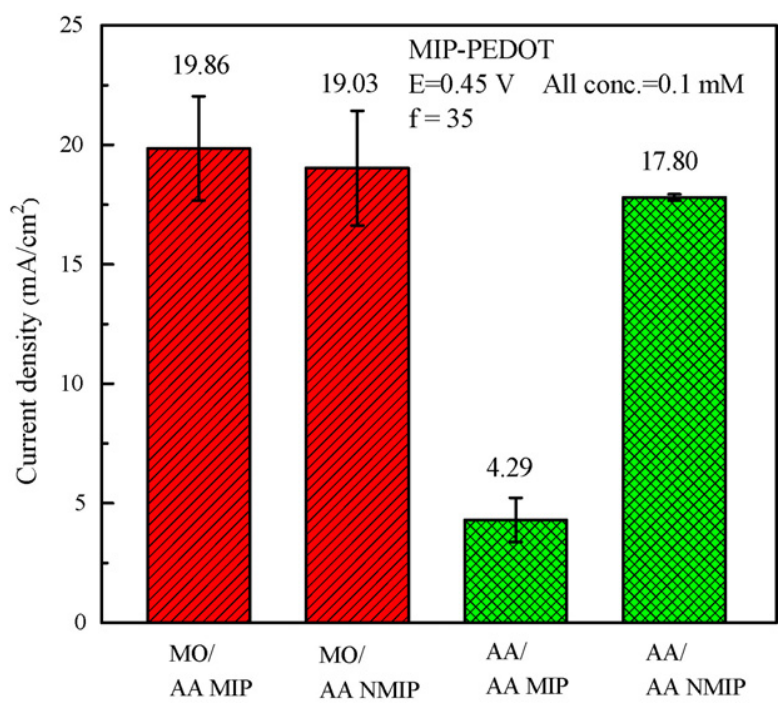

Fig. 6. The steady-state current densities of MO or AA collected on the MIP-PEDOT microelectrode after flowing $0.1 \mathrm{mM}$ MO or $0.1 \mathrm{mM}$ AA over the AA MIP-Nylon 6 and AA NMIP-Nylon 6 pre-separators. All other experimental conditions are kept the same as those described in Fig. 5.

that morphine's molecular imprinting sites were formed during electropolymerization of the MIP-PEDOT electrode. Since the noise of the system was $18.8 \mathrm{nA} / \mathrm{cm}^{2}$, the detection limit was calculated around $0.3 \mu \mathrm{M}$ at a signal-to-ratio $(\mathrm{S} / \mathrm{N})$ of 3 for the MIP-PEDOT electrode.

The response time of the sensing electrode also was an important parameter. As expected, the response time of our sensing device decreased upon miniaturization. The major advantage of the integrated chip comprised with multiple micro-pump and morphine-sensing electrodes was its enhanced pumping rate. Therefore, the sample transport time was reduced and the response was correspondingly enhanced. Most current changes occur within the first $25 \mathrm{~s}$, and there was almost no change in the following $100 \mathrm{~s}$. For example, for $0.1 \mathrm{mM}$ morphine, the response time was around $22 \mathrm{~s}$ (95\% of saturation level). The microelectrodes were washed with PBS after sensing. The recovery time was measured to be $91.6 \mathrm{~s}$.

Another issue for morphine sensing was the interference from other compositions existing in the samples. For example, the interference caused by the AA in human blood could not be ignored. Thus their influence on the detector must be removed. In order to minimize the AA interference, AA MIP-Nylon 6 and AA NMIP-Nylon 6 were placed in the upstream of the microchannel as a pre-separator to adsorb AA before sensing morphine. The steady-state current densities, collected with the MIP-PEDOT modified electrode, which was placed in the downstream of the microchannel, by flowing $0.1 \mathrm{mM}$ morphine or $0.1 \mathrm{mM}$ AA over the AA MIP-Nylon 6 and AA NMIP-Nylon 6, were shown in Fig. 6. The reproducibility of the data was verified three times as indicated by the error bars. Two slash bars represent the current densities recorded after flowing $0.1 \mathrm{mM}$ morphine over the AA MIP-Nylon 6 and AA NMIP-Nylon 6 pre-reactors (denoted as MO/AA MIP and MO/AA NMIP); two grid bars represent the current densities collected after flowing $0.1 \mathrm{mM}$ AA over the AA MIP-Nylon
6 and AA NMIP-Nylon 6 pre-separators (denoted as AA/AA MIP and AA/AA NMIP). The current densities only decreased slightly with the uses of the MO/AA MIP and MO/AA NMIP (slash bar) pre-separators, as compared to those without using pre-separators. This implied that both the AA nylon-MIP and AA nylon-NMIP pre-separators could not adsorb morphine. On the other hand, as shown in the grid bar, AA was captured and adsorbed by the AA nylon-MIP pre-separator, which was placed in the upstream of the microchannel. This results indicated that AA adsorption in the pre-separator region reduced the interference effect of AA and enhance the sensitivity for the detection of morphine.

\section{Conclusion}

This paper presents a microfluidic chip capable of automatically performing precise and continuous morphine measurements. The novel combination of microfluidics, MIP, and electrochemical techniques provides a promising system for morphine detection. Using microfluidic technology, a precise amount of samples inside multiple microchannels is transported to the sensing zone using micropumps/microvalves. Experimental data show that the developed morphine-MIP microfluidic system successfully detects morphine with concentrations ranging from 0.01 to $0.2 \mathrm{mM}$. While compared to large-scale instruments, the developed microfluidic system is compact in size, and consumes fewer samples, decreasing the required sample volume from milliliters to microliters. More importantly, the whole process can be performed automatically.

\section{Acknowledgements}

The authors would like to thank partial financial supports from the National Science Council in Taiwan (NSC 94-3112-B006-002) and the MOE Program for Promoting Academic Excellence of Universities (EX-91-E-FA09-5-4). Access of major fabrication equipment from Center for Micro/Nano Technology Research, National Cheng Kung University is also greatly appreciated.

\section{References}

[1] H.M. Lee, C.W. Lee, Determination of morphine and codeine in blood and bile by gas chromatography with a derivation procedure, J. Anal. Toxicol. 15 (1991) 182-187.

[2] G. Chari, A. Gulati, R. Bhat, I.R. Tebbett, High-performance liquid chromatographic determination of morphine, morphine-3-glucuronide, morphine-6-glucuronide and codeine in biological samples using multiwavelength forward optical detection, J. Chromatogr. 571 (1991) 263-270.

[3] F. Tagliaro, D. Franchi, R. Dorizzi, M. Marigo, High-performance liquid chromatographic determination of morphine in biological samples: an overview of separation methods and detection techniques, J. Chromatogr. 488 (1989) 215-228.

[4] M.E. Soares, V. Seabra, M.L. Bastos, Comparative study of different extractive procedures to quantify morphine in urine by HPLC-UV, J. Liq. Chromatogr. 15 (1992) 1533-1541.

[5] J.G. Guillot, M. Lefebvre, J.P. Weber, Determination of heroin, 6acetylmorphine, and morphine in biological fluids using their propionyl derivatives with ion trap GC-MS, J. Anal. Toxicol. 21 (1997) 127-133. 
[6] R. Dams, T. Benijts, W.E. Lambert, A.P. De Leenheer, Simultaneous determination of in total 17 opium alkaloids and opioids in blood and urine by fast liquid chromatography-diode-array detection-fluorescence detection, after solid-phase extraction, J. Chromatogr. B 773 (2002) 53-61.

[7] S.W. Lewis, P.S. Francis, K.F. Lim, G.E. Jenkins, X.D. Wang, Pulsed flow chemistry: a new approach to solution handling for flow analysis coupled with chemiluminescence detection, Analyst 125 (2000) 1869-1874.

[8] G. Sakai, K. Ogata, T. Uda, N. Miura, N. Yamazoe, A surface plasma resonance-based immunosensor for highly sensitive detection of morphine, Sens. Actuators B: Chem. 49 (1998) 5-12.

[9] F. Xu, M. Gao, L. Wang, T. Zhou, L. Jin, J. Jin, Amperometric determination of morphine on cobalt hexacyanoferrate modified electrode in rat brain microdialysates, Talanta 58 (2002) 427-432.

[10] A.I. Bouquillon, D. Freeman, D.E. Moulin, Simultaneous solid-phase extraction and chromatographic analysis of morphine and hydromorhpine in plasma by high-performance liquid chromatography with electrochemical detection, J. Chromatogr. 577 (1992) 354-357.

[11] M.E. Eldefrawi, N.L. Azer, N. Nath, N.A. Anis, M.S. Bangalore, K.P. O'Connell, R.P. Schwartz, J. Wright, A sensitive solid-phase fluoroimmunoassay for detection of opiates in urine, Appl. Biochem. Biotechnol. 87 (2000) 25-35.

[12] L. Ye, K. Mosbach, Molecularly imprinted microspheres as antibody binding mimics, React. Funct. Polym. 48 (2001) 149-157.

[13] T.C. Chou, K.M. Ng, S.H. Wang, Gold-solid polymer electrolyte sensor for detecting dissolved oxygen in water, Sens. Actuators B: Chem. 66 (2000) 184-186.

[14] W.J. Liaw, S.T. Ho, J.J. Wang, O.Y.P. Hu, J.H. Li, Determination of morphine by high-performance liquid chromatography with electrochemical detection: application to human and rabbit pharmacokinetic studies, J. Chromatogr. B 714 (1998) 237-245.

[15] A.J. Cunningham, Introduction to Bioanalytical Sensors, Wiley, New York, 1998, p. 267.

[16] B.A. Rashid, G.W. Aherne, M.F. Katmeh, P. Kwasowski, D. Stevenson, Determination of morphine in urine by solid-phase immunoextraction and high-performance liquid chromatography with electrochemical detection, J. Chromatogr. A 797 (1998) 245-250.

[17] A.W.E. Wright, J. Watt, M. Kennedy, T. Cramond, M.T. Smith, Quantification of morphine, morphine-3-glucuronide, and morphine-6-glucuronide in plasma and cerebrospinal-fluid using solid-phase extraction and highperformance liquid-chromatography with electrochemical detection, Ther. Drug Monit. 16 (1994) 200-208.

[18] R.J. Ansell, D. Kriz, K. Mosbach, Molecularly imprinted polymers for bioanalysis: chromatography, binding assays and biomimetic sensors, Curr. Opin. Biotechnol. 7 (1996) 89-94.

[19] H.C. Hsu, L.C. Chen, K.C. Ho, Colorimetric detection of morphine in a molecularly imprinted polymer using an aqueous mixture of $\mathrm{Fe}^{3+}$ and $\left[\mathrm{Fe}(\mathrm{CN})_{6}\right]^{3-}$, Anal. Chim. Acta 504 (2004) 141-147.

[20] D. Kriz, K. Mosbach, Competitive amperometric morphine sensor based on an agarose immobilized molecularly imprinted polymer, Anal. Chim. Acta 300 (1995) 71-75.

[21] E. Reid, H.M. Hill, I.D. Wilson, Drug Development Assay Approaches Including Molecular Imprinting and Biomarkers, The Royal Society of Chemistry, Cambridge, UK, 1998, pp. 28-36.

[22] K.C. Ho, W.M. Yeh, T.S. Tung, J.Y. Liao, Amperometric detection of morphine based on poly(3,4-ethylenedioxythiophene) immobilized molecularly imprinted polymer particles prepared by precipitation polymerization, Anal. Chim. Acta 542 (2005) 90-96.

[23] A.J. Bard, L.R. Faulkner, Electrochemical Methods: Fundamentals and Applications, 2nd ed., John Wiley and Sons, New York, 2001.
[24] C.H. Wang, G.B. Lee, Automatic bio-sensing diagnostic chips integrated with micro-pumps and micro-valves for multiple disease detection, Biosens. Bioelectron. 21 (2005) 419-425.

[25] K. Haupt, K. Mosbach, Molecularly imprinted polymers and their use in biomimetic sensors, Chem. Rev. 100 (2000) 2495-2501.

[26] W.M. Yeh, K.C. Ho, Amperometric morphine sensing using a molecularly imprinted polymer-modified electrode, Anal. Chim. Acta 542 (2005) $76-82$.

[27] M.R. Moelleer, S. Steinmeyer, T. Kraemer, Determination of drugs abuse in blood, J. Chromatogr. B 713 (1998) 91-109.

[28] P.P. Reddy, T. Kobayashi, M. Abe, N. Fujii, Molecular imprinted Nylon-6 as a recognition material of amino acid, Eur. Polym. J. 38 (2002) 521-529.

\section{Biographies}

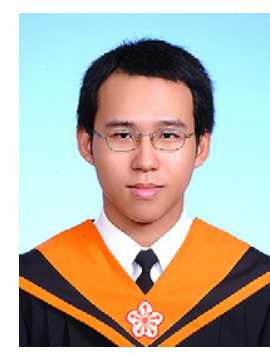

Chen-Hsun Weng received his B.S. degree in Department of Civil Engineering from National Taiwan University in 2004. He is currently a graduate student in Department of Engineering Science at National Cheng Kung University. His research interests are MEMS, microfluidics, and bio-sensing.

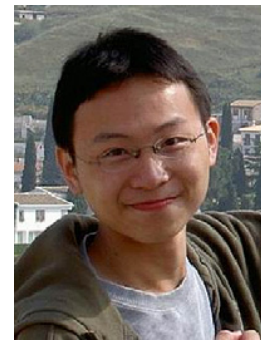

Wei-Ming Yeh received his B.S. degree in Department of Chemical Engineering from National Chung Hsing University, Taiwan in 2003. He received M.S. degree in Chemical Engineering from National Taiwan University in 2005. Currently, he is in the army to fulfill the compulsory military service. His research interest mainly is focused on molecularly imprinted polymers and their applications to bio-sensors.

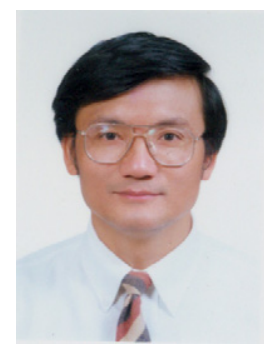

Kuo-Chuan Ho received his B.S. and M.S. degrees in Department of Chemical Engineering from National Cheng Kung University, Taiwan in 1978 and 1980, respectively. He received his Ph.D. in Chemical Engineering at the University of Rochester, USA in 1986. Currently he is a Professor jointly appointed by the Department of Chemical Engineering and Institute of Polymer Science and Engineering at National Taiwan University. His research interests mainly surround applications of chemically modified electrodes on sensing and electro-optical devices.

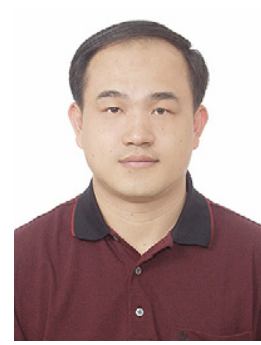

Gwo-Bin Lee received his B.S. and M.S. degrees in Department of Mechanical Engineering from National Taiwan University in 1989 and 1991, respectively. He received his Ph.D. in Mechanical and Aerospace Engineering from University of California, Los Angeles, USA in 1998. He is currently a Professor in the Department of Engineering Science at National Cheng Kung University. His research interests lie on microfluidics, bio-sensing, nanobiotechnology and its biomedical applications. 